\title{
Impact of the COVID-19 lockdown in France on the diagnosis and staging of breast cancers in a tertiary cancer centre
}

\author{
Pierre-Antoine Linck ${ }^{1}$. Cassandre Garnier ${ }^{1}$. Marie-Pierre Depetiteville ${ }^{1}$ - Gaëtan MacGrogan ${ }^{2}$. \\ Simone Mathoulin-Pélissier ${ }^{3,4,5} \cdot$ Nathalie Quénel-Tueux $^{6} \cdot$ Hélène Charitansky $^{7}$ - Martine Boisserie-Lacroix ${ }^{1}$. \\ Foucauld Chamming's ${ }^{1}$
}

Received: 29 March 2021 / Revised: 9 July 2021 / Accepted: 23 July 2021 / Published online: 13 October 2021

(c) European Society of Radiology 2021

\begin{abstract}
Objectives Due to COVID-19, a lockdown took place between March 17 and May 1, 2020, in France. This study evaluates the impact of the lockdown on the diagnosis and staging of breast cancers in a tertiary cancer centre.

Methods Our database was searched for all consecutive invasive breast cancers diagnosed in our institution during the lockdown (36 working days), during equivalent periods of 36 working days before and after lockdown and a reference period in 2019. The number and staging of breast cancers diagnosed during and after lockdown were compared to the pre-lockdown and reference periods. Tumour maximum diameters were compared using the Mann-Whitney test. Proportions of tumour size categories $(\mathrm{T})$, ipsilateral axillary lymph node invasion $(\mathrm{N})$ and presence of distant metastasis $(\mathrm{M})$ were compared using Fisher's exact test.

Results Compared to the reference period ( $n=40$ in average), the number of breast cancers diagnosed during lockdown $(n=32)$ decreased by $20 \%$ but increased by $48 \%$ after the lockdown $(n=59)$. After the lockdown, comparatively to the reference period, breast cancers were more often symptomatic $(86 \%$ vs $57 \%$; $p=0.001)$ and demonstrated bigger tumour sizes $(p=0.0008)$, the rates of small tumours (T1) were reduced by $38 \%$, locally advanced cancers (T3, T4) increased by $80 \%$ and lymph node invasion increased by $64 \%$.

Conclusion The COVID-19 lockdown was associated with a $20 \%$ decrease in the number of diagnosed breast cancers. Because of delayed diagnosis, breast cancers detected after the lockdown had poorer prognosis with bigger tumour sizes and higher rates of node invasion.

\section{Key Points}

- The number of breast cancer diagnosed in a large tertiary cancer centre in France decreased by $20 \%$ during the first COVID-19 lockdown.

- Because of delayed diagnosis, breast cancers demonstrated bigger tumour size and more frequent axillary lymph node invasion after the lockdown.

- In case of a new lockdown, breast screening programme and follow-up examinations should not be suspended and patients with clinical symptoms should be encouraged to seek attention promptly.
\end{abstract}

Keywords COVID-19 $\cdot$ Lockdown $\cdot$ Breast cancer $\cdot$ Diagnosis $\cdot$ Neoplasm staging

Foucauld Chamming's

f.chammings@bordeaux.unicancer.fr

1 Department of Radiology, Institut Bergonié, Comprehensive Cancer Centre, 33076 Bordeaux, France

2 Department of Pathology, Institut Bergonié, Comprehensive Cancer Centre, 33076 Bordeaux, France

3 Unité D'Épidémiologie Et de Recherche Cliniques, Institut Bergonié, Comprehensive Cancer Centre, 33076 Bordeaux, France
4 Inserm UMR 1219, Epicene Team, Bordeaux, France

5 Inserm CIC-EC 1401, Bordeaux, France

6 Department of Medical Oncology, Institut Bergonié, Comprehensive Cancer Centre, 33076 Bordeaux, France

7 Department of Surgery, Institut Bergonié, Comprehensive Cancer Centre, 33076 Bordeaux, France 


\section{Abbreviations \\ COVID-19 Coronavirus disease 2019 \\ DBT Digital breast tomosynthesis \\ MRI Magnetic resonance imaging}

\section{Introduction}

The coronavirus disease 2019 (COVID-19) outbreak was associated with $0.3-1.5 \%$ mortality in Western countries, predominantly in elderly people and in those with comorbidities such as obesity, diabetes mellitus or arterial hypertension [1-3]. To mitigate the spread of the virus and to avoid overload of the health care system, most countries announced a lockdown in early 2020 to limit interpersonal physical interactions. In several developed countries, nonurgent medical consultations and imaging examinations were cancelled or postponed to protect potentially at-risk patients from developing a severe form of COVID, preserve health care system facilities and reduce physical interactions between health workers and patients. Like what was first set-up in China, management processes were adapted in radiology departments in France to avoid cross-infections [4].

According to the national French and European Breast Oncological and Imaging Society's recommendations [5, 6], breast screening programmes were suspended and follow-up examinations in patients with a personal history of breast cancer were postponed. After the start of the lockdown, only diagnostic examinations were maintained in patients who had clinical or radiological abnormal findings. However, it was observed that many patients with urgent or severe diseases also deferred medical consultation either because they were anxious about the COVID-19 risk or because they believed health care facilities would not be accessible [6-9]. In particular, a decline in the number of patients with a newly diagnosed cancer was reported during the COVID19 pandemic [10].

With regard to breast cancer, a recent publication confirmed that, as a result of the suspension of the screening programme, the number of breast cancers diagnosed decreased during the lockdown in The Netherlands [11] . A recently published modelling study estimated that delay in diagnosis could increase by 7.9-9.6\% the number of breast cancer-related deaths in England [12]. However, these recent publications are only modelling studies and there is a lack of data on the real impact of the COVID-19 pandemic on the staging of breast cancers actually diagnosed during and after the lockdown. In this context, the objective of our study was to evaluate the impact of the lockdown in France on the diagnosis and staging of breast cancers diagnosed in a tertiary cancer centre.

\section{Materials and methods}

Institutional Ethics Review Board approval was obtained for this retrospective study and informed consent was waived.

\section{Lockdown and pre-/post-lockdown period (Fig. 1)}

In France, the first lockdown took place from March 17 to May 11, 2020 (8 weeks or 36 working days). Following the announcement of the lockdown, most screening and followup examinations were very quickly postponed in public and private imaging centres and rescheduled several weeks later. When the lockdown ended, screening was reopened and women could normally undergo their planned follow-up examinations. To evaluate the impact of the lockdown on the diagnosis of breast cancers, we defined a pre-lockdown period of 36 working days prior to the lockdown (January 27 to March 16, 2020) and a post-lockdown period of 36 working days immediately following the lockdown (May 11 to July 1, 2020). For comparison, we defined three corresponding periods in 2019, similar in terms of date range (January 28-July 3, 2019) and number of working days (36 days). However, the activity of the breast-imaging unit during these periods of 36 days may be variably impacted by some factors such as public holidays. In order to use a more representative period of our routine activity for comparison, we therefore chose to pool the three periods of 36 days in a single period of 108 days to be used as a reference.

\section{Patient selection}

We searched our institutional database for all consecutive patients who underwent an imaging-guided percutaneous breast biopsy performed under US, digital breast tomosynthesis (DBT) or MRI during the lockdown, the pre- and postlockdown periods and during the reference period in 2019. All patients with invasive cancer on pathology findings were eligible for inclusion in this retrospective study. Patients who underwent biopsy of an axillary lymph node or of a lesion outside the breast were not included. Exclusion criteria were as follows: on pathology patients with benign findings, pure in situ carcinoma without invasive components and invasive cancers other than breast carcinomas; patients with local recurrence after ipsilateral radical mastectomy (for which no follow-up imaging examination is recommended) and invasive carcinomas that were not the index malignant lesion (additional lesions). Because we aimed to evaluate locoregional staging of breast cancers diagnosed in our institution, patients who had a biopsy of the index lesion outside our centre were also excluded from analysis. 

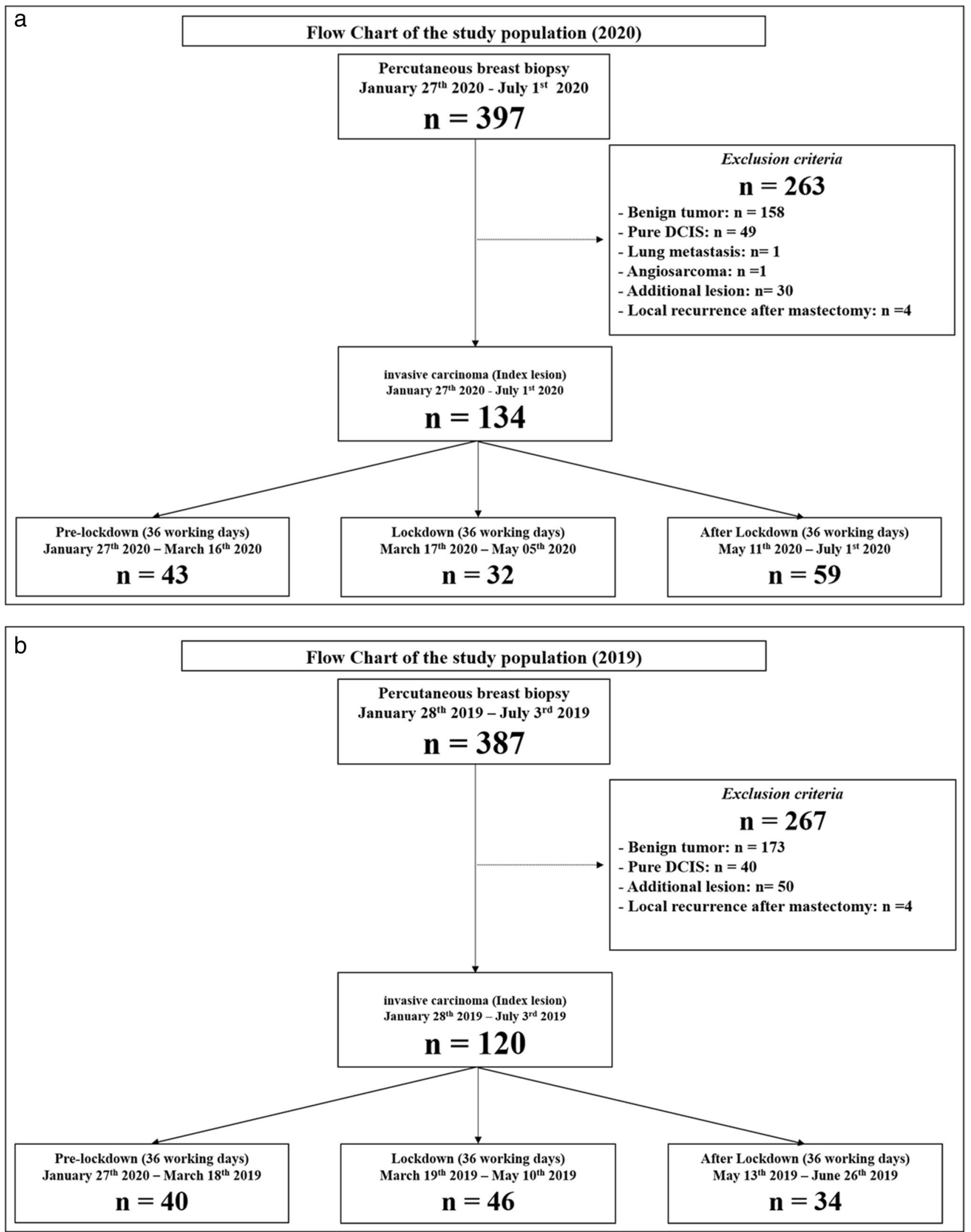

Fig. 1 Flowchart of the study population. a Number of invasive breast cancers diagnosed in in our institution before, during and after the 2020 COVID-19 lockdown $(3 \times 36=108$ working days $)$. b Number of invasive breast cancers diagnosed in our institution between January 28 and July 3, 2019 (108 working days). DCIS, ductal carcinoma in situ; IDC, invasive ductal carcinoma 


\section{Statistical analysis}

The clinical parameters collected were age and presence of a clinical symptom such as a palpable mass, nipple or skin retraction, nipple discharge or inflammatory breast at the time of diagnosis. To evaluate tumour extension according to the $8^{\text {th }}$ edition of the tumour, lymph node and metastasis (TNM) classification [13], we collected index tumour maximum diameter, presence of axillary lymph node invasion and presence of distant metastasis at the time of diagnosis. When available, tumour size was assessed using the maximum diameter of the invasive component on the surgical specimen. For patients where tumour size could not be assessed on the surgical specimen, patients who underwent neoadjuvant chemotherapy or neoadjuvant hormonotherapy or patients for whom delay between diagnosis and surgery was longer than 1 month, the tumour maximum diameter was determined on imaging. Because our objective was to evaluate extension of invasive carcinomas, extension of microcalcifications was not taken into consideration when evaluating the tumour size.

Axillary lymph node status was evaluated either on surgical findings (either sentinel node biopsy of axillary dissection) or on imaging results in cases where patients underwent neoadjuvant treatment. Tumour maximum diameters are reported as median with interquartile range (IQR) in square brackets. Age is reported as mean with standard deviation in brackets. Maximum tumour diameters were compared using the Mann-Whitney test. Proportions of tumour size categories $(\mathrm{T})$, node invasion $(\mathrm{N})$ and presence of distant metastasis (M) were compared between the different periods using Fisher's exact test for non-parametric data. Differences in proportions of tumour size categories and axillary lymph nodes invasions across the studied periods are reported as relative changes. $p \leq 0.05$ was considered to indicate a statistically significant difference. Statistical analysis was performed using software JMP, version 12, SAS institute.

\section{Results}

\section{Number of cancers}

Between January 27 and July 1, 2020 (108 working days), 134 primary invasive breast carcinomas were diagnosed in 134 women (mean age: $64+/-16$ years) in our institution (Fig. 1). Comparatively, 120 primary invasive cancers were detected in 120 women (mean age: $66+/-14$ years) during the reference period of 2019. In 2020, 43 invasive cancers were diagnosed during the pre-lockdown period, 32 during lockdown and 59 during the post-lockdown period. In average in 2019 , we detected 40 invasive cancers per corresponding period of 36 working days (Table 1). In comparison with the pre-lockdown and the reference period of 2019 , the number of cancers diagnosed in our institution respectively decreased by $26 \%$ and $20 \%$ during the lockdown and increased by $37 \%$ and $48 \%$ during the post-lockdown period (Fig. 2).

\section{Tumour staging (Table 1)}

\section{Proportion of symptomatic cancers}

Breast cancers diagnosed during lockdown were symptomatic in 75\% (24/32) of cases, versus 57\% (68/108) in 2019 $(p=0.07)$ and $47 \%(20 / 43)$ before lockdown $(p=0.02)$. Breast cancers detected after the lockdown were significantly

Table 1 Number and staging of invasive breast cancers diagnosed before, during and after the lockdown

\begin{tabular}{lllll}
\hline & 2019 & Pre- lockdown 2020 & Lockdown 2020 & Post-lockdown 2020 \\
\hline & $N(\%)$ & $N(\%)$ & $N(\%)$ & $N(\%)$ \\
Working days & 108 & 36 & 36 & 36 \\
Number of cancers & 40 & 43 & 32 & 59 \\
(average per 36 working days) & & & \\
Maximum diameter (mm) [IQR] & $18[10 ; 30]$ & $15[9 ; 25]$ & $23[15 ; 40]$ & $25[16 ; 40]$ \\
Presence of a clinical symptom & $68(57)$ & $20(47)$ & $24(75)$ & $51(86)$ \\
T1 & $66(55)$ & $29(67)$ & $13(40)$ & $20(34)$ \\
T2 & $37(31)$ & $13(30)$ & $13(40)$ & $23(39)$ \\
T3 & $9(8)$ & $1(2)$ & $5(16)$ & $9(15)$ \\
T4 & $8(7)$ & $0(0)$ & $16(50)$ & $7(12)$ \\
N+ & $39(33)$ & $12(28)$ & $3(9)$ & $32(54)$ \\
M+ & $3(3)$ & $2(5)$ & $5(8)$ \\
\hline
\end{tabular}

Tumour maximum diameters are indicated as median with interquartile range $[I Q R]$ in square brackets. T1, primary tumour maximum diameter $\leq 20 \mathrm{~mm}$; T2, tumour maximum diameter $>20 \mathrm{~mm}$ and $\leq 50 \mathrm{~mm}$; T3, tumour maximum diameter $>50 \mathrm{~mm}$; T4, direct extension to chest wall and/or to the skin; $\mathrm{N}+$, ipsilateral axillary lymph node invasion; $\mathrm{M}+$, distant metastasis 
Fig. 2 Graph showing the number of cancers diagnosed in our institution days before, during and after the 2020 COVID-19 lockdown, compared to the average number of cancers diagnosed per equivalent period of 36 days in 2019
๖ำ

Number of cancers

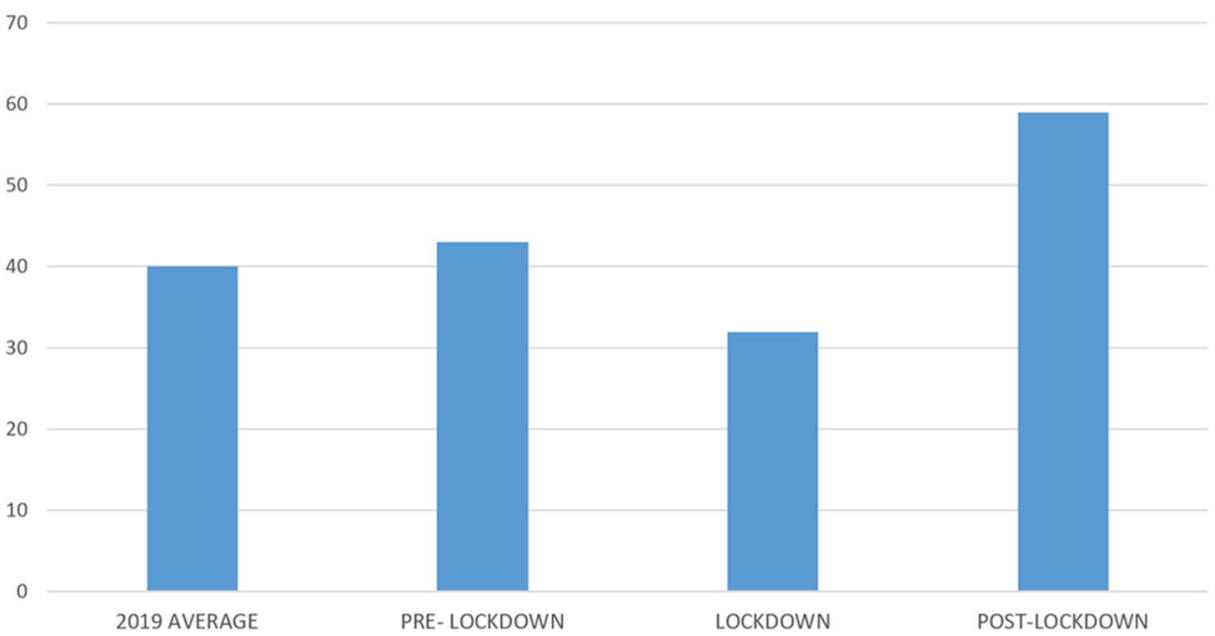

more often symptomatic $(86 \%(51 / 59)$ of cases) than in 2019 and in the pre-lockdown period $(p<0.0001)$.

\section{Tumour size (T)}

Tumour maximum diameter was assessed radiologically in $61 \%$ (155/254) and pathologically in 39\% (99/254) of cases.

In 2020 , the median maximum diameter of the tumour was $18 \mathrm{~mm}$ (IQR: 10; 30) in the reference period of 2019 , $15 \mathrm{~mm}$ (IQR: 9; 25) before lockdown, $23 \mathrm{~mm}$ (IQR: 15; 40) during lockdown and $25 \mathrm{~mm}$ (IQR: 16; 40) after lockdown. Maximum tumour diameter was significantly bigger during lockdown and post-lockdown periods in comparison with the reference period of 2019 ( $p=0.002$ and $p=0.0002$ respectively) and before lockdown ( $p=0.02$ and $p=0.0008$ respectively). After the lockdown, the proportion of small tumours (T1) was lower than in the reference period of 2019
$(-38 \%, p=0.01)$ while the proportion of locally advanced cancers $(\mathrm{T} 3, \mathrm{~T} 4)$ was higher $(+80 \%, p=0.04)$ (Fig. 3).

\section{Axillary node invasion (Fig. 4)}

After lockdown, the proportion of ipsilateral axillary lymph node invasion was $64 \%$ higher than that in the reference period of $2019(p=0.006)$.

\section{Presence of distant metastasis}

Presence of distant metastasis at the time of diagnosis tended towards a higher frequency after lockdown when compared to the reference period of 2019 , but this difference did not reach significance ( $8 \%$ versus $3 \%, p=0.12$ ).
Fig. 3 Proportion of tumour size categories (according to the TNM classification) in the reference period of 2019, and before, during and after the 2020 COVID-19 lockdown

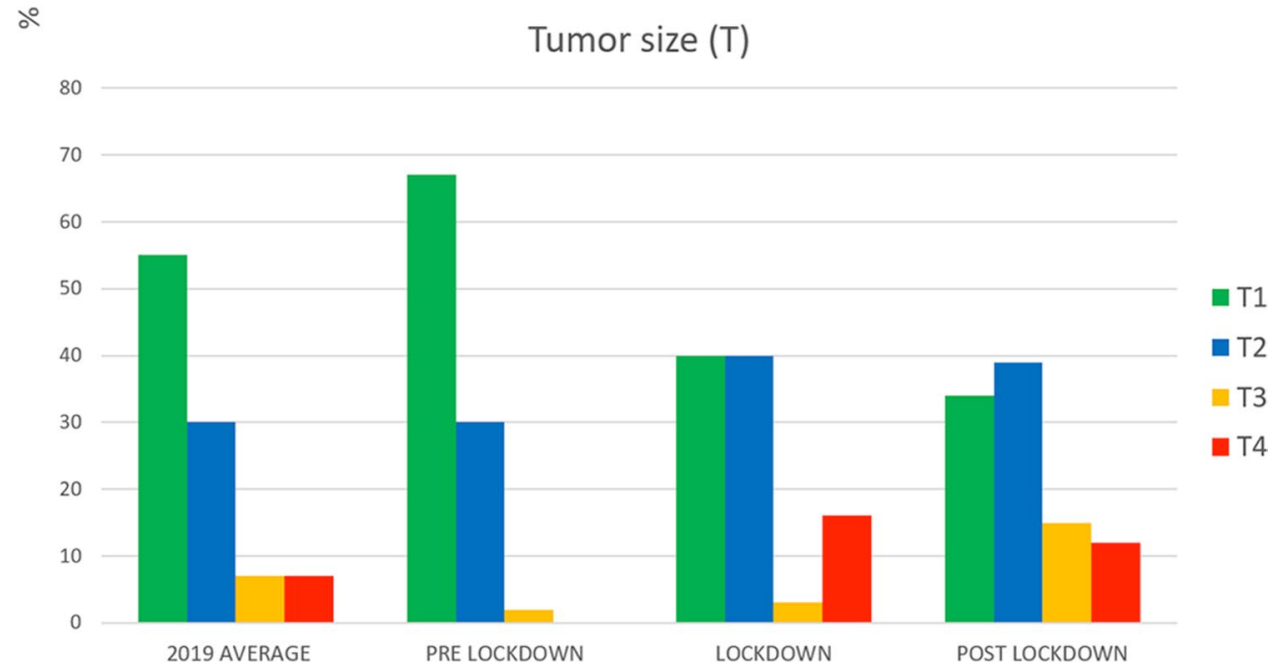


Fig. 4 Rate of ipsilateral axillary lymph node involvement in the reference period of 2019 , and before, during and after the 2020 COVID-19 lockdown
๖ำ

Axillary node invasion (N)

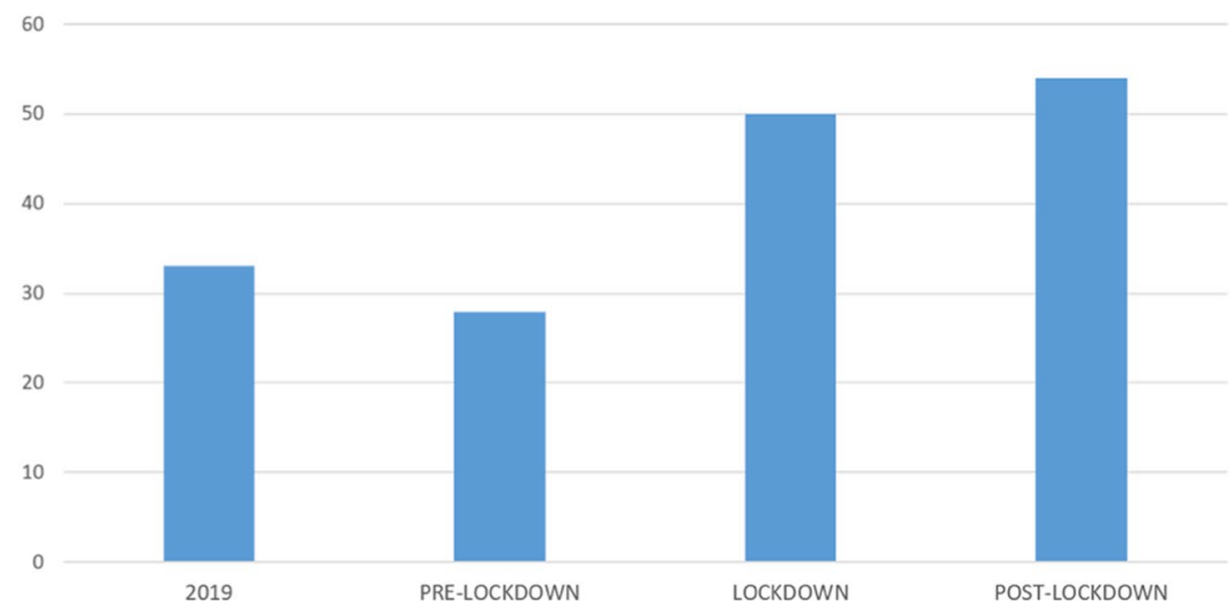

\section{Discussion}

During the first COVID-19 lockdown in France, following National and European recommendations, breast cancer screening programmes were suspended and breast imaging examinations were limited to patients with clinical symptoms or abnormal findings on imaging [5, 14]. Most followup examinations in patients with a personal history of breast cancer were also postponed. As a consequence, the number of invasive breast cancers diagnosed in our institution decreased during the lockdown period. Concomitantly, they were more often palpable, demonstrated bigger tumour sizes and were associated with a higher rate of axillary lymph node invasion. This can be explained by the fact that, probably because of the interruption of the screening programme, we diagnosed more cancers that were associated with clinical symptoms, which are more likely to be more advanced tumours.

After lockdown, patients were once again encouraged to participate in the breast cancer screening programme or undergo imaging follow-up [15]. We observed that the number of invasive breast cancers diagnosed in our institution increased again, and was reaching levels that were higher than during the immediate pre-lockdown period. This finding suggests that the cancers undiagnosed during the lockdown were eventually 'caught-up' during the immediate post-lockdown period. During the post-lockdown period, invasive breast cancers were more often palpable, and were detected at a later stage. In particular, frequency of locally advanced tumours (T3 and T4) and axillary lymph node invasion $(\mathrm{N})$, which are known to be the most important prognostic factors for long-term survival [16] respectively increased by $80 \%$ and 64\% in comparison with those in 2019. Given that screening programmes and follow-up examinations had resumed at this time, and that the lower number of cancers diagnosed during the lockdown was balanced by the increasing detection of cases in the post-lockdown period, we conclude that the later stage of breast cancers was not only related to the selection of more advanced cases but was also due to a delayed diagnosis. As the case shown in Fig. 5, it was not only screening mammograms and follow-up examinations that were postponed for several weeks during lockdown, but some patients with clinical symptoms such as palpable masses were reported to postpone their clinical consultations and/or imaging work-up during the lockdown due to virus- or facility-related concerns, despite the fact that the hospital was open and clinical and radiological facilities available for consultations. It is well known that a delay in diagnosis and treatment may significantly impact the outcome of cancers [17-21]. Our study confirms that the delay in breast cancer diagnosis induced by the first lockdown in France actually had an impact on cancer staging and prognosis. Our results are of paramount interest because they highlight the collateral damage resulting from a severe lockdown in which access to medical consultations is limited. They may encourage imaging societies and public health authorities to recommend that breast imaging examinations including screening and follow-up mammograms would not be suspended in case of a new lockdown. Moreover, women should be informed to seek medical attention as soon as possible in case of clinical symptoms, such as presence of a palpable mass.

Our results are in line with what has been reported by other recent studies. Like ours, several studies have observed a large decrease of cancer-related patient encounters across Europe [21, 22]. A significant reduction in the number of breast screening examinations [10] and number of breast cancers diagnosed has been reported. However, as in our study, Dinmohammed et al. observed that the number of diagnoses ultimately reached once the post-lockdown 'catch up' occurred was approximately at the level of the expected values [11]. To the best of our knowledge, our 

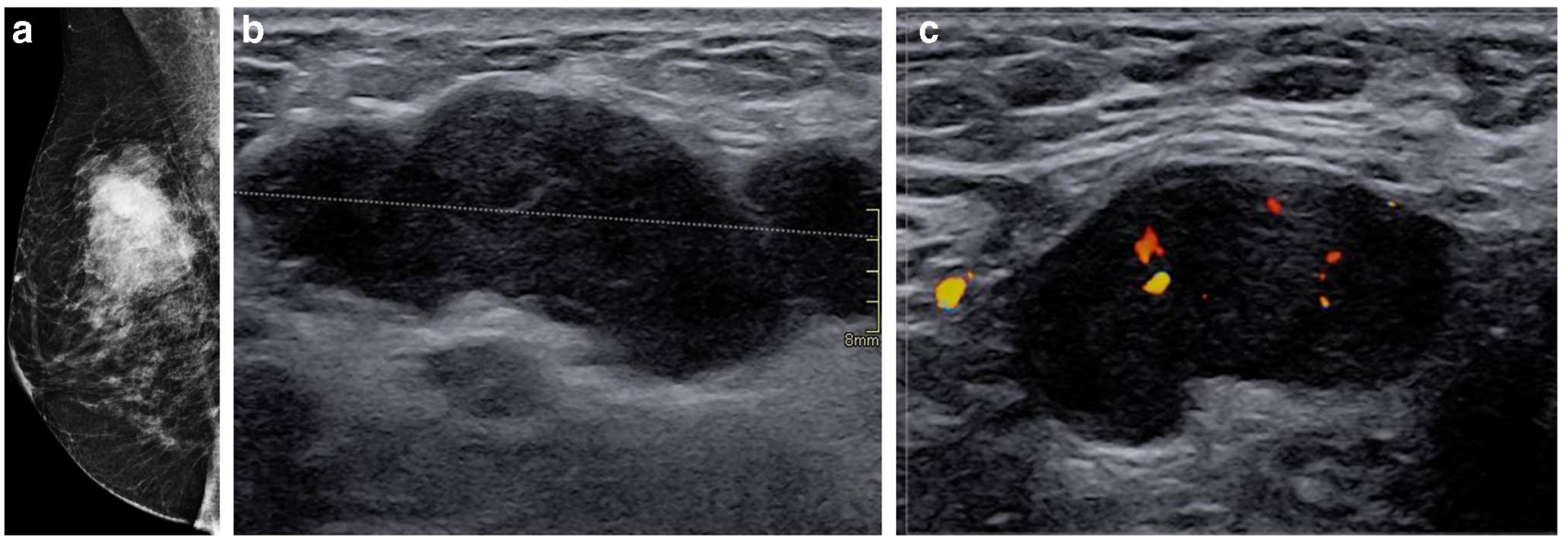

Fig. 5 Right medio-lateral oblique mammogram (a) and breast (b) and axillary (c) ultrasound (US) in a 53-year-old woman showing a 51-mm (T3) palpable mass of the upper-outer aspect of the right breast with enlarged ipsilateral axillary lymph node. The patient had a palpable mass since the end of February 2020 but delayed her con-

study is the first to confirm that the delay in breast cancer diagnoses or cancellations and postponements induced by the COVID-19 crisis has resulted in more advanced disease at diagnosis.

Our study has some limitations. First, we only evaluated the impact of the lockdown on the diagnosis of breast cancer in one institution, albeit a large one. Our results therefore are not necessarily applicable to other centres and while our tertiary cancer centre is a good reflection of the impact of the lockdown on breast cancer diagnosis in our country, multicentre studies would be needed to validate our results on a national scale. Secondly, we only evaluated the breast cancers that were diagnosed in our institution and not those that underwent percutaneous biopsies outside our hospital. It is possible that this would have induced a selection bias. However, our method for patient selection was identical for all studied periods allowing comparability. Third, histological verification of lymph nodes was not available for all the cases that were defined as positive. Thus, overestimation of positive lymph node status cannot really be excluded. Fourth, since the decrease of the number of cancers diagnosed during the lockdown could be due to multiple reasons (such as suspension of screening and follow-up examinations, and also to postponement of diagnostic work-up by symptomatic patients), we could not identify one single explanation to the results of our study. In particular, we could not clearly determine the special role of the suspension of the screening on our findings. Further studies based on data derived from the national organized screening programme would therefore be of great interest to identify the real impact of the suspension of the screening during the lockdown on breast cancer diagnosis. Fifth, our study might have a selection bias towards more aggressive cases that sultation because of the COVID-19 outbreak. She finally underwent a US-guided biopsy on May 29, 2020, after the end of the lockdown revealing a triple-negative invasive carcinoma of non-specific type (NST) with axillary lymph node invasion

seek attention in a tertiary care facility, compared to less aggressive ones that might have preferred smaller facilities. Finally, our study was conducted too early after the lockdown to be able to evaluate its impact on recurrence and mortality rates. In a recent population-based modelling study, the potential impact of the delayed diagnosis on prognosis of breast cancers has been estimated suggesting that it could induce an increase of 7.9 to $9.6 \%$ of breast cancer-related deaths [12]. However, future studies would also be of great interest to evaluate the real delayed impact of the lockdown on mortality and recurrence rates of breast cancers.

In conclusion, our study shows that the lockdown due to the COVID-19 pandemic was associated with a $20 \%$ decrease in the number of diagnosed breast cancers during lockdown, but increased diagnoses immediately after the lockdown 'caught up' for this reduction. Because of delayed diagnosis, breast cancers detected after the lockdown had poorer prognosis with a greater tumour size and higher rate of node involvement, strongly suggesting that, in case of a new lockdown, breast screening programme and follow-up examinations should not be suspended, and patients should be informed to seek medical attention as soon as possible in case of clinical symptoms.

Acknowledgements The authors thank Pippa McKelvie-Sebileau for medical editing in English.

Funding The authors state that this work has not received any funding.

\section{Declarations}

Guarantor The scientific guarantor of this publication is Foucauld Chamming's. 
Conflict of interest The authors of this manuscript declare no relationships with any companies, whose products or services may be related to the subject matter of the article.

Statistics and biometry One of the authors has significant statistical expertise.

Informed consent Written informed consent was waived by the Institutional Review Board.

Ethical approval Institutional Review Board approval was obtained.

\section{Methodology}

- Retrospective

- Observational

- Performed at one institution

\section{References}

1. Zhou Y, Yang Q, Chi J et al (2020) Comorbidities and the risk of severe or fatal outcomes associated with coronavirus disease 2019: a systematic review and meta-analysis. Int J Infect Dis 99:47-56. https://doi.org/10.1016/j.ijid.2020.07.029

2. Grover A, Oberoi M (2020) A systematic review and meta-analysis to evaluate the clinical outcomes in COVID-19 patients on angiotensin-converting enzyme inhibitors or angiotensin receptor blockers. Eur Heart J Cardiovasc Pharmacother. https://doi.org/ 10.1093/ehjcvp/pvaa064

3. Weiss P, Murdoch DR (2020) Clinical course and mortality risk of severe COVID-19. Lancet 395:1014-1015. https://doi.org/10. 1016/S0140-6736(20)30633-4

4. Gutzeit A, Li Q, Matoori S et al (2020) What can European radiologists learn from the outbreak of COVID-19 in China? A discussion with a radiologist from Wuhan. Eur Radiol 30:3609-3611. https://doi.org/10.1007/s00330-020-06841-6

5. Gligorov J, Bachelot T, Pierga J-Y et al (2020) COVID-19 and people followed for breast cancer: French guidelines for clinical practice of Nice-St Paul de Vence, in collaboration with the Collège Nationale des Gynécologues et Obstétriciens Français (CNGOF), the Société d'Imagerie de la Femme (SIFEM), the Société Française de Chirurgie Oncologique (SFCO), the Société Française de Sénologie et Pathologie Mammaire (SFSPM) and the French Breast Cancer Intergroup-UNICANCER (UCBG). Bull Cancer 107:528-537. https://doi.org/10.1016/j.bulcan.2020.03. 008

6. Dietz JR, Moran MS, Isakoff SJ et al (2020) Recommendations for prioritization, treatment, and triage of breast cancer patients during the COVID-19 pandemic. The COVID-19 pandemic breast cancer consortium. Breast Cancer Res Treat 181:487-497. https:// doi.org/10.1007/s10549-020-05644-Z

7. Aboukaïs R, Devalckeneer A, Boussemart P et al (2020) Impact of COVID-19 pandemic on patients with intracranial aneurysm rupture. Clin Neurol Neurosurg 201:106425. https://doi.org/10. 1016/j.clineuro.2020.106425

8. Tapper EB, Asrani SK (2020) The COVID-19 pandemic will have a long-lasting impact on the quality of cirrhosis care. J Hepatol 73:441-445. https://doi.org/10.1016/j.jhep.2020.04.005
9. Kaufman HW, Chen Z, Niles J, Fesko Y (2020) Changes in the number of US patients with newly identified cancer before and during the coronavirus disease 2019 (COVID-19) pandemic. JAMA Netw Open 3:e2017267. https://doi.org/10.1001/jaman etworkopen.2020.17267

10. Maringe C, Spicer J, Morris M et al (2020) The impact of the COVID-19 pandemic on cancer deaths due to delays in diagnosis in England, UK: a national, population-based, modelling study. Lancet Oncol 21:1023-1034. https://doi.org/10.1016/S14702045(20)30388-0

11. Hortobagyi GN, Connolly JL, D’Orsi CJ et al (2017) American Joint Committee on Cancer (AJCC) cancer staging manual . 8th edn. In: ew York : Springer. pp 589-28

12. Ceugnart L, Delaloge S, Balleyguier C et al (2020) Breast cancer screening and diagnosis at the end of the COVID-19 confinement period, practical aspects and prioritization rules: recommendations of 6 French health professionals societies. Bull Cancer 107:623-628. https://doi.org/10.1016/j.bulcan.2020.04.006

13. Soerjomataram I, Louwman MWJ, Ribot JG et al (2008) An overview of prognostic factors for long-term survivors of breast cancer. Breast Cancer Res Treat 107:309-330. https://doi.org/10. 1007/s10549-007-9556-1

14. Neal RD, Tharmanathan P, France B et al (2015) Is increased time to diagnosis and treatment in symptomatic cancer associated with poorer outcomes? Systematic review. Br J Cancer 112(Suppl 1):S92-107. https://doi.org/10.1038/bjc.2015.48

15. Vose JM (2020) Delay in cancer screening and diagnosis during the COVID-19 pandemic: what is the cost? Oncology (Williston Park) 34:343. https://doi.org/10.46883/ONC.2020.3409.0343

16. Miranda DLP, Nogueira-Rodrigues A, Fagundes TP et al (2020) COVID-19 threatens to cause collateral delay in cancer diagnosis. Sao Paulo Med J 138:347-348. https://doi.org/10.1590/15163180.2020.033730062020

17. Ricci F, Fania L, Paradisi A et al (2020) Delayed melanoma diagnosis in the COVID-19 era: increased Breslow thickness in primary melanomas seen after the COVID-19 lockdown. J Eur Acad Dermatol Venereol. https://doi.org/10.1111/jdv.16874

18. Sud A, Jones ME, Broggio J et al (2020) Collateral damage: the impact on outcomes from cancer surgery of the COVID-19 pandemic. Ann Oncol 31:1065-1074. https://doi.org/10.1016/j. annonc.2020.05.009

19. Aviran E, Laks S, Benvenisti H et al (2020) The impact of the COVID-19 pandemic on general surgery acute admissions and urgent operations: a comparative prospective study. Isr Med Assoc J 11:673-679

20. Dinmohamed AG, Cellamare M, Visser O et al (2020) The impact of the temporary suspension of national cancer screening programmes due to the COVID-19 epidemic on the diagnosis of breast and colorectal cancer in the Netherlands. J Hematol Oncol 13:147. https://doi.org/10.1186/s13045-020-00984-1

21. de Azambuja E, Trapani D, Loibl S, et al (2020) ESMO Management and treatment adapted recommendations in the COVID-19 era: breast cancer. ESMO Open 5https://doi.org/10.1136/esmoo pen-2020-000793

22. London JW, Fazio-Eynullayeva E, Palchuk MB et al (2020) Effects of the COVID-19 pandemic on cancer-related patient encounters. JCO Clin Cancer Inform 4:657-665. https://doi.org/10.1200/CCI. 20.00068

Publisher's note Springer Nature remains neutral with regard to jurisdictional claims in published maps and institutional affiliations. 